\title{
On a Nonlocal Damping Model in Ferromagnetism
}

\author{
M. Moumni ${ }^{1,2}$ and M. Tilioua ${ }^{2}$ \\ ${ }^{1}$ MIA CNRS EA 3165, Université de La Rochelle, 17000 La Rochelle, France \\ ${ }^{2}$ FST Errachidia, Laboratoire M2I, Equipe MAMCS, Université My Ismaïl, BP 509, Boutalamine, 52000 Errachidia, Morocco
}

Correspondence should be addressed to M. Tilioua; tilioua@melix.org

Received 15 April 2015; Accepted 12 July 2015

Academic Editor: Wei Wu

Copyright ( $) 2015$ M. Moumni and M. Tilioua. This is an open access article distributed under the Creative Commons Attribution License, which permits unrestricted use, distribution, and reproduction in any medium, provided the original work is properly cited.

We consider a mathematical model describing nonlocal damping in magnetization dynamics. The model consists of a modified form of the Landau-Lifshitz-Gilbert (LLG) equation for the evolution of the magnetization vector in a rigid ferromagnet. We give a global existence result and characterize the long time behaviour of the obtained solutions. The sensitivity of the model with respect to large and small nonlocal damping parameters is also discussed.

\section{Introduction and Preliminary Results}

In this work, we are dealing with a mathematical model arising in the theory of magnetization dynamics with nonlocal damping. We will consider the model proposed by Nembach et al. [1]. It is given by a modified LLG equation. The modification lies in the presence of a second-order space derivative of magnetization in the effective field. To describe the model equations, we consider $\Omega \subset \mathbb{R}^{3}$ a bounded and regular open set of $\mathbb{R}^{3}$. The generic point of $\mathbb{R}^{3}$ is denoted by $x$. We assume that a ferromagnetic material occupies the domain $\Omega$.

In what follows, $S^{2}$ represents the unit sphere of $\mathbb{R}^{3}$. The magnetization field of the ferromagnetic material which belongs to $S^{2}$ almost everywhere is denoted by $M(t, x)$. Its evolution is governed by the following modified LLG equation (see $[1,2])$ :

$$
\frac{1}{1+\alpha^{2}}\left(\partial_{t} M-\alpha M \times \partial_{t} M\right)=-M \times \mathscr{H}(M)
$$

$$
\text { in } Q=(0, T) \times \Omega \text {, }
$$

subject to initial conditions

$$
M(0, x)=M_{0}(x) \quad \text { in } \Omega
$$

and boundary conditions

$$
M \times\left(D \partial_{\nu} M+\zeta \partial_{\nu} \partial_{t} M\right)=0 \quad \text { on }(0, T) \times \partial \Omega,
$$

where the symbol $\times$ denotes the vector cross product in $\mathbb{R}^{3}$. The positive constant $\alpha$ represents the damping parameter. The effective magnetic field $\mathscr{H}$ depends on $M$ and is given by

$$
\mathscr{H}(M)=D \Delta M+\zeta \Delta \partial_{t} M+\phi(M)+\mathscr{H}_{\mathrm{dem}}(M) .
$$

The first term on the right-hand side of (4) is called the exchange magnetic field, where the positive constant $D$ is the exchange coefficient and the term parameterized by the positive constant $\zeta$ describes nonlocal damping in ferromagnets. The $\zeta$-term was introduced in [2]; see also [1]. The third term is the bulk anisotropy field (which generally is taken as linear with respect to $M$ ), and $\mathscr{H}_{\text {dem }}$ is the demagnetizing field, satisfying in $(0, T) \times \mathbb{R}^{3}$ the stray field equation

$$
\begin{aligned}
\operatorname{div}\left(\mathscr{H}_{\mathrm{dem}}+\bar{M}\right) & =0, \\
\operatorname{curl} \mathscr{H}_{\mathrm{dem}} & =0,
\end{aligned}
$$

where $\bar{M}$ is the extension by 0 of $M$ outside the magnetic domain $\Omega$.

Notice that, for the sake of simplicity, the bulk uniaxial anisotropy field, generally taken as linear in $M$, and the magnetostatic field which satisfy the stray field equation are 
not considered since they only induce more computations and have no mathematical influence on the results we obtain.

We define the energy

$$
\mathscr{E}(t)=D \int_{\Omega}|\nabla M|^{2} \mathrm{~d} x .
$$

The following energy estimate holds.

Lemma 1. If $M$ is a solution of problem (1), then it satisfies, at least formally, the energy estimate

$$
\begin{aligned}
& \frac{\mathrm{d}}{\mathrm{d} t} \mathscr{E}(t)+2 \zeta \int_{\Omega}\left|\nabla \partial_{t} M\right|^{2} \mathrm{~d} x+\frac{2 \alpha}{1+\alpha^{2}} \int_{\Omega}\left|\partial_{t} M\right|^{2} \mathrm{~d} x \\
& \quad=0 .
\end{aligned}
$$

Proof. The techniques to obtain (7) are analogous to those used, for example, in $[3,4]$. We rewrite LLG equation (1) in the following form:

$$
\begin{aligned}
& \left(\alpha \partial_{t} M-\left(1+\alpha^{2}\right) \mathscr{H}\right) \\
& \quad=\alpha M \times\left(\alpha \partial_{t} M-\left(1+\alpha^{2}\right) \mathscr{H}\right)-\left(1+\alpha^{2}\right) \mathscr{H} .
\end{aligned}
$$

Multiplying (8) by $\left(\alpha \partial_{t} M-\left(1+\alpha^{2}\right) \mathscr{H}\right)$ and using the saturation constraint $|M|^{2}=1$ almost everywhere yields to

$$
\frac{\alpha}{1+\alpha^{2}}\left|\partial_{t} M\right|^{2}=\mathscr{H} \cdot \partial_{t} M
$$

Integrating (9) on $\Omega$, the right-hand side of (9) becomes

$$
\begin{aligned}
\int_{\Omega} \mathscr{H} \cdot \partial_{t} M \mathrm{~d} x= & -D \int_{\Omega} \partial_{i} M \partial_{i} \partial_{t} M \mathrm{~d} x \\
& -\zeta \int_{\Omega} \partial_{i} \partial_{t} M \partial_{i} \partial_{t} M \mathrm{~d} x \\
& +\int_{\partial \Omega}\left(D \partial_{\nu} M+\zeta \partial_{\nu} \partial_{t} M\right) \partial_{t} M \mathrm{~d} \sigma,
\end{aligned}
$$

where we have used the summation convention that repeated indices are summed over and $\partial_{i}$ denotes the derivative with respect to space variable $x_{i}$. Converting the two last terms of the right-hand side of (10) allows us to get (7).

Before dealing with problem (1), let us first review some previous results. We limit ourselves to mentioning a handful of references concerning existence and we refer to the survey [5] for a more detailed bibliographical account. The general framework (although without nonlocal damping, i.e., the case $\zeta=0$ ) has been established in earlier papers (see, for instance, $[6,7]$ ) using Faedo-Galerkin/Penalization (FGP) method. This method gives an approximated sequence of solutions converging to a global solution of the problem. Next results concern systems with further dissipation terms. For example, the modification considered in [8] consists in adding to the standard dissipation term in the LLG equation another higher-order term of the type $\Delta^{2} M$. The FGP method is also used to solve the problem. In [9], a model with dry-friction dissipation, which is accounted by adding a dry-friction-like term to the standard Gilbert damping, is studied. Let us mention that a model of ferromagnetic material with hysteresis effects is studied in [10]. In this model, the magnetic moment behaviour is described by the nonlinear Landau-Lifshitz equation with an additional term modeling the hysteresis. This term takes the form of a maximal monotone operator acting on the time derivative of the magnetic moment. For this relaxed model, local existence of regular solutions is proved. Note that in the framework of current-induced magnetization dynamics the work [11] addresses global existence of weak solutions to a LLG equation where a transport term is added to effective field taking into account the effect of the injected current. A model of magnetization switching with inertial effects modeled by means of a second-order time derivative term in the effective field is considered in [12] where existence of weak solutions and their long time behaviour were established. All these proofs are based on some penalization and using various kind of regularizations.

To state the existence result, we start with the definition of weak solutions to problem (1).

Definition 2. Given that $M_{0} \in \mathbb{H}^{1}(\Omega)$ such that $\left|M_{0}\right|=1$ almost everywhere in $\Omega$ we call $M(t, x)$ a weak solution to LLG equation (1) if

(1) for all $T>0, M \in L^{\infty}\left((0, T) ; \mathbb{H}^{1}(\Omega)\right), \partial_{t} M \in$ $L^{2}\left((0, T) ; \mathbb{L}^{2}(\Omega)\right) \cap L^{\infty}\left((0, T) ; \mathbb{L}^{2}(\Omega)\right)$, and $M$ satisfies the saturation constraint $|M(t, x)|=1$ for almost everywhere in $\mathbb{R}^{+} \times \Omega$;

(2) $M(0, \cdot)=M_{0}(\cdot)$ in the trace sense;

(3) for all $G \in \mathbb{M}^{1}(Q) \cap \mathscr{C}_{0}(Q)$, there holds

$$
\begin{aligned}
& \frac{1}{1+\alpha^{2}} \int_{Q}\left(\partial_{t} M-\alpha M \times \partial_{t} M\right) \cdot G \mathrm{~d} x \mathrm{~d} t \\
& =D \int_{Q} M \times \partial_{i} M \cdot \partial_{i} G \mathrm{~d} x \mathrm{~d} t \\
& \quad+\zeta \int_{Q} M \times \partial_{i} \partial_{t} M \cdot \partial_{i} G \mathrm{~d} x \mathrm{~d} t
\end{aligned}
$$

(4) for all $t \geq 0$, we have

$$
\begin{aligned}
\mathscr{E}(t) & +2 \zeta \int_{0}^{t} \int_{\Omega}\left|\nabla \partial_{t} M\right|^{2} \mathrm{~d} x \\
& +\frac{2 \alpha}{1+\alpha^{2}} \int_{0}^{t} \int_{\Omega}\left|\partial_{t} M\right|^{2} \mathrm{~d} x \mathrm{~d} t \leq \mathscr{E}(0),
\end{aligned}
$$

where $\mathscr{E}(t)$ is given by (6)

We have the following global existence result.

Theorem 3. Let $M_{0} \in \mathbb{H}^{1}(\Omega)$ be such that $\left|M_{0}(x)\right|^{2}=1$ almost everywhere. Then there exists a global weak solution $M$ of problem (1) in the sense of Definition 2.

Proof. The proof follows a standard scheme (Faedo-Galerkin method with a penalization of the saturation constraint) as usual in general Landau-Lifshitz-Gilbert equation; see [6, 7, 13]. 
The rest of the paper is divided as follows. In the next section we investigate the long time behaviour of the solutions. Section 3 discusses the sensitivity of the obtained solutions with respect to nonlocal damping factor $\zeta$. More precisely we characterize the limit problem for both high and small $\zeta$. We conclude the paper in Section 4 by giving some comments.

\section{The Limit as $t$ Goes to $+\infty$}

We investigate the long time behaviour of the solutions. More precisely, we study the $\omega$-limit set of the trajectories and characterize the $\omega$-limit points as solutions of a suitable stationary problem. We proceed as in Carbou-Fabrie [13].

Let $M$ be a weak solution of (1). We call $\omega$-limit set of the trajectory $M$ the following set:

$$
\begin{aligned}
& \omega(M)=\left\{\mathbf{m} \in \mathbb{U}^{1}(\Omega), \exists t_{n}, \lim _{n \rightarrow+\infty} t_{n}=+\infty, M\left(t_{n}, \cdot\right)\right. \\
& \left.\rightarrow \mathbf{m} \text { in } \mathbb{T}^{1}(\Omega) \text { weakly }\right\} .
\end{aligned}
$$

Consider a weak solution $M$ of (1). From the energy estimate (12), the $\omega$-limit set $\omega(M)$ is nonempty. We denote by $\mathbf{m}$ a point of this set. There exists a nondecreasing sequence $\left(t_{n}\right)_{n}$ such that $t_{n} \rightarrow+\infty$ and $M\left(t_{n}, \cdot\right) \rightarrow \mathbf{m}$ in $\mathbb{M}^{1}(\Omega)$ weakly. Since $\Omega$ is a smooth bounded domain, then $M\left(t_{n}, \cdot\right)$ tends to $\mathbf{m}$ in $\mathbb{L}^{p}(\Omega)$ strongly for $p \in[1,6[$,and extracting a subsequence, we assume that $M\left(t_{n}, \cdot\right)$ tends to $\mathbf{m}$ almost everywhere, so that the saturation constraint $|\mathbf{m}|=1$ is satisfied almost everywhere. In addition, we remark that, for all $n$, $\left|M\left(t_{n}, \cdot\right)\right|=1$ almost everywhere, so that $\left\|M\left(t_{n}, \cdot\right)\right\|_{L^{\infty}(\Omega)}=1$. By interpolation inequalities in $\mathbb{L}^{p}$ spaces, we obtain that, for all $p<+\infty, M\left(t_{n}, \cdot\right)$ tends to $\mathbf{m}$ in $\mathbb{L}^{p}(\Omega)$ strongly.

For $s \in(-1,1)$ and $x \in \Omega$ we define for $n$ large enough

$$
m_{n}(s, x)=M\left(t_{n}+s, x\right) .
$$

We have the following convergence result.

Lemma 4. The sequence $\left(m_{n}\right)_{n \geq 1}$ satisfies the following convergences:

$$
\begin{aligned}
& m_{n} \longrightarrow \mathbf{m} \quad \text { in } \mathbb{L}^{2}((-1,1) \times \Omega) \text { strongly, } \\
& m_{n} \rightarrow \mathbf{m} \quad \text { in } \mathbb{L}^{2}\left((-1,1) ; \mathbb{T}^{1}(\Omega)\right) \text { weakly. }
\end{aligned}
$$

Proof. Following [14], we have the estimate

$$
\begin{gathered}
\frac{1}{2} \int_{-1}^{1} \int_{\Omega}\left|m_{n}(s, x)-M\left(t_{n}, x\right)\right|^{2} \mathrm{~d} x \mathrm{~d} s \\
\leq \int_{t_{n}-1}^{+\infty} \int_{\Omega}\left|\partial_{t} M(\tau, x)\right|^{2} \mathrm{~d} x \mathrm{~d} \tau .
\end{gathered}
$$

As $\partial_{t} M$ lies in $\mathbb{L}^{2}\left(\mathbb{R}^{+} \times \Omega\right)$, one gets

$$
\lim _{n \rightarrow+\infty} \frac{1}{2} \int_{-1}^{1} \int_{\Omega}\left|m_{n}(s, x)-M\left(t_{n}, s\right)\right|^{2} \mathrm{~d} x \mathrm{~d} s=0 .
$$

Since $M\left(t_{n}, \cdot\right)$ tends to $\mathbf{m}$ in $\mathbb{L}^{2}(\Omega)$ strongly, $m_{n}$ tend to $\mathbf{m}$ in $\mathbb{L}^{2}\left((-1,1) ; \mathbb{L}^{2}(\Omega)\right)$ strongly. Moreover, we have obviously seen that $\left(\nabla m_{n}\right)_{n \geq 1}$ is bounded in $\mathbb{L}^{2}((-1,1) \times \Omega)$ so there exists a subsequence still denoted by $\left(m_{n}\right)_{n \geq 1}$ such that $m_{n}$ tends to $\mathbf{m}$ in $\mathbb{L}^{2}\left((-1,1), \mathbb{T}^{1}(\Omega)\right)$ weakly and in $\mathbb{L}^{2}\left((-1,1), \mathbb{L}^{2}(\Omega)\right)$ strongly almost everywhere in $(-1,1) \times \Omega$. This ends the proof of the lemma.

Now, we consider a function $\rho \in \mathscr{C}_{0}^{\infty}((-1,1))$ such that $0 \leq \rho(\tau) \leq 1,\left|\rho^{\prime}(\tau)\right| \leq 2$. In the weak formulation (11) we take as test function $G(t, x)=\rho\left(t-t_{n}\right) \Psi(x)$, where $\Psi$ is a function lying in $\mathscr{D}(\bar{\Omega})$ and $n$ fixed. We obtain after the change of variables $s=t-t_{n}$

$$
\begin{aligned}
& \frac{1}{1+\alpha^{2}}\left(\int_{-1}^{1} \int_{\Omega}\left(\partial_{t} m_{n}(s, x)-\alpha m_{n}(s, x) \times \partial_{t} m_{n}(s, x)\right)\right. \\
& . \Psi(x) \rho(s) \mathrm{d} x \mathrm{~d} s)-D \int_{-1}^{1} \int_{\Omega} m_{n}(s, x) \\
& \times \partial_{i} m_{n}(s, x) \cdot \partial_{i} \Psi(x) \rho(s) \mathrm{d} x \mathrm{~d} s \\
& -\zeta \int_{-1}^{1} \int_{\Omega} m_{n}(s, x) \times \partial_{i}\left(\partial_{t} m_{n}\right)(s, x) \cdot \partial_{i} \Psi(x) \rho(s) \mathrm{d} x \mathrm{~d} s \\
& =0 .
\end{aligned}
$$

We take the limit of the previous equation when $n$ tends to $+\infty$. In order to pass to the limit, we bound each term of the above formulation. For example, for the last term we have

$$
\begin{aligned}
& \left|\int_{-1}^{1} \int_{\Omega} m_{n}(s, x) \times \partial_{i} \partial_{t} m_{n}(s, x) \cdot \partial_{i} \Psi(x) \rho(s) \mathrm{d} x \mathrm{~d} s\right| \\
& \quad \leq \int_{-1}^{1} \int_{\Omega}\left|m_{n}(s, x) \times \partial_{i} \partial_{t} m_{n}(s, x)\right|\left|\partial_{i} \Psi(x)\right| \\
& \quad \cdot|\rho(s)| \mathrm{d} x \mathrm{~d} s \leq \int_{-1}^{1} \int_{\Omega} \mid m_{n}(s, x) \\
& \quad \times \partial_{i} \partial_{t} m_{n}(s, x)|| \partial_{i} \Psi(x) \mid \mathrm{d} x \mathrm{~d} s \\
& \quad \leq\left(\int_{-1}^{1} \int_{\Omega}\left|m_{n}(s, x) \times \partial_{i} \partial_{t} m_{n}(s, x)\right|^{2} \mathrm{~d} x \mathrm{~d} s\right)^{1 / 2} \\
& \quad \cdot\left(\int_{-1}^{1} \int_{\Omega}\left|\partial_{i} \Psi(x)\right|^{2} \mathrm{~d} x \mathrm{~d} s\right)^{1 / 2} \\
& \quad \leq\left(\int_{-1}^{1} \int_{\Omega}\left|\partial_{i} \partial_{t} m_{n}(s, x)\right|^{2} \mathrm{~d} x \mathrm{~d} s\right)^{1 / 2} \\
& \quad\left(\int_{\Omega}\left|\partial_{i} \Psi(x)\right|^{2} \mathrm{~d} x\right)^{1 / 2} \cdot \\
& \quad \cdot\left(\int_{-1}^{1} \int_{\Omega}\left|\partial_{i} \Psi(x)\right|^{2} \mathrm{~d} x \mathrm{~d} s\right)^{1 / 2} \\
& \left.\quad \int_{2}^{t_{n}+1} \int_{t_{n}-1}\left|\partial_{i} \partial_{t} M(s, x)\right|^{2} \mathrm{~d} x \mathrm{~d} s\right)^{1 / 2}
\end{aligned}
$$


Since $\nabla \partial_{t} M$ belongs to $\mathbb{L}^{2}\left(\mathbb{R}^{+} \times \Omega\right)$, this last term tends to zero as $n$ goes to $+\infty$. In the same way we pass to the limit in the other terms to obtain

$$
\int_{-1}^{1} \rho(s) \mathrm{d} s \int_{\Omega} \mathbf{m}(x) \times \partial_{i} \mathbf{m}(x) \cdot \partial_{i} \Psi(x) \mathrm{d} x=0,
$$

which implies

$$
\int_{\Omega} \mathbf{m}(x) \times \partial_{i} \mathbf{m}(x) \cdot \partial_{i} \Psi(x) \mathrm{d} x=0
$$

for all $\Psi \in \mathscr{D}(\bar{\Omega})$.

We proved the following.

Theorem 5. If $M$ is a weak solution of (1), then each point $\mathbf{m}$ in $\omega(M)$ is a weak solution of the steady state system

$$
\mathbf{m} \in \mathbb{H}^{1}(\Omega) ; \quad|\mathbf{m}|=1 \text {, almost everywhere }
$$

$\mathbf{m} \times \Delta \mathbf{m}=0 \quad$ in $\Omega$

which should be understood in the weak sense (21).

Remark 6. The solutions of (22) are known as harmonic maps to the unit sphere, which turn up in several equations in physics, such as Ginzburg-Landau equation, and were extensively investigated mathematically in the past decades due to their importance both in mathematics and in many applied fields; see, for example, $[15,16]$. For the links between harmonic maps and the solutions of the LLG equation we refer to the book [17, Chapter 4]. Some results on the dynamic version of (22) can be found in [18].

\section{Limiting Process for High and Small Nonlocal Damping}

We first analyze the limiting process when $\zeta$ goes to $+\infty$. We denote by $M^{\zeta}$ a global weak solution of LLG equation (1) associated with $\zeta$ and the initial data $M_{0}$ and satisfying the energy inequality (7). We have the estimates.

Lemma 7. There exists $C>0$ independent of $\zeta$ such that the sequence $M^{\zeta}$ satisfies the estimates

$$
\begin{aligned}
\left|M^{\zeta}(t, x)\right|^{2} & =1 \text { almost everywhere, } \\
\left|\nabla M^{\zeta}\right|_{L^{\infty}\left(\mathbb{R}^{+} ; L^{2}(\Omega)\right)} & \leq C \\
\left|\nabla \partial_{t} M^{\zeta}\right|_{L^{2}\left(\mathbb{R}^{+} ; L^{2}(\Omega)\right)} & \leq C \zeta^{-1} \\
\left|\partial_{t} M^{\zeta}\right|_{L^{2}\left(\mathbb{R}^{+} ; L^{2}(\Omega)\right)} & \leq C
\end{aligned}
$$

We also have the following.

Lemma 8. The sequence $M^{\zeta}$ is compact in $L_{\text {loc }}^{2}\left(\mathbb{R}^{+} ; L^{2}(\Omega)\right)$.

Lemmas 7 and 8 imply the following convergence results.
Lemma 9. There exists a subsequence still denoted by $m^{\zeta}$ such that

$$
\begin{gathered}
M^{\zeta} \rightarrow M \text { weakly-* } \quad \text { in } L^{\infty}\left(\mathbb{R}^{+} ; \mathbb{H}^{1}(\Omega)\right), \\
\partial_{t} M^{\zeta} \rightarrow \partial_{t} M \text { weakly } \quad \text { in } L^{2}\left(\mathbb{R}^{+} ; \mathbb{H}^{-1}(\Omega)\right), \\
\nabla M^{\zeta} \rightarrow \nabla M \text { weakly } \quad \text { in } L^{2}\left(\mathbb{R}^{+} ; \mathbb{L}^{2}(\Omega)\right), \\
\nabla \partial_{t} M^{\zeta} \longrightarrow 0 \text { strongly in } L_{\mathrm{loc}}^{2}\left(\mathbb{R}^{+} ; \mathbb{L}^{2}(\Omega)\right), \\
\partial_{t} M^{\zeta} \longrightarrow 0 \text { strongly in } L_{\mathrm{loc}}^{2}\left(\mathbb{R}^{+} ; \mathbb{L}^{2}(\Omega)\right), \\
M^{\zeta} \longrightarrow M \text { strongly } \quad \text { in } L_{\mathrm{loc}}^{2}\left(\mathbb{R}^{+} ; \mathbb{L}^{2}(\Omega)\right) .
\end{gathered}
$$

Moreover, $M$ satisfies the saturation condition $|M|=1$.

The above convergences allow one to conclude.

Theorem 10. Let $M$ be the limit of a subsequence of $M^{\zeta}$ as $\zeta$ goes to $+\infty$. Then, the domain $\Omega$ is uniformly magnetized.

Remark 11. The result of Theorem 10 is of interest. In fact, the added term in the effective field can act as a control for magnetization switching.

Our aim now is to pass to the limit as $\zeta$ goes to zero in (1). We denote by $M^{\zeta}$ a global weak solution of LLG equation (1) associated with $\zeta$ and the initial data $M_{0}$ and satisfying the energy inequality (7). The bound on $\sqrt{\zeta} \nabla \partial_{t} M^{\zeta}$ in $L^{2}\left(0, T ; \mathbb{L}^{2}(\Omega)\right)$ (see (7)) allows one to get the following.

Theorem 12. Let $M$ be the limit of a subsequence of $M^{\zeta}$. Then, $M$ satisfies

$$
\begin{aligned}
& \partial_{t} M-\alpha M \times \partial_{t} M=-\left(1+\alpha^{2}\right) M \times(D \Delta M) \\
& \text { in } \mathbb{R}^{+} \times \Omega \\
& M(0)=M_{0} \quad \text { in } \Omega, \\
& M \times D \partial_{n} M=0 \quad \text { on } \partial \Omega \text {. }
\end{aligned}
$$

Moreover, $M$ satisfies the saturation condition $|M(t, x)|=1$ almost everywhere.

Remark 13. From (25), we can say that, for lower values of $\zeta$, the magnetic nonlocal damping vanishes and the classical switching continues for ever. In other words, the magnetization precesses several times around the effective field direction before it reaches equilibrium.

\section{Concluding Remarks}

In this paper, we have considered nonlocal damping in magnetization dynamics. The model consists of a generalized LLG equation that contains a term characterizing nonlocal damping expressed in terms of $\Delta \partial_{t} M$ in the effective field. The long time behaviour of the solutions is characterized and the sensitivity of the model to nonlocal damping parameter 
$\zeta$ is discussed. The results obtained can be applied without difficulty to the case of effective field with anisotropy and demagnetizing fields. Note that the model considered in this paper neglects the additional damping due to transversal spin currents whose form is $\partial_{i} M \times \partial_{i} \partial_{t} M$. In this case the LLG equation takes the following form (see Nembach et al. [1]):

$$
\begin{aligned}
\partial_{t} M-\alpha M \times \partial_{t} M= & -\left(1+\alpha^{2}\right) M \\
& \times\left(D \Delta M+\zeta \Delta \partial_{t} M\right)+\zeta \partial_{i} M \\
& \times \partial_{i} \partial_{t} M .
\end{aligned}
$$

Interestingly, the additional damping cannot be written in terms of the free energy, and therefore it cannot be derived from the functional derivative of the free energy with respect to the local magnetization. This new term can significantly change the domain-wall structure in ferromagnetic materials. It would be interesting to consider this problem from both the theoretical and the numerical points of view. In particular, global existence of weak solutions will require more detailed studies.

We finally mention that an important progress was done to design schemes constructing the weak solutions to the general LLG equation. Several schemes were proposed and their convergence to weak solutions was proved. A significant step forward in the convergence theory of numerical schemes has been done recently; see [19-21]. This will be helpful to give a strategy for efficient computer implementation which may reflect the true nature of the augmentation of the LLG model considered in this paper.

\section{Conflict of Interests}

The authors declare that there is no conflict of interests regarding the publication of this paper.

\section{Acknowledgments}

This work was supported by the PHC Volubilis program MA/14/301 "Elaboration et analyse de modèles asymptotiques en micro-magnétisme, magnéto-élasticité et électroélasticité" with joint financial support from the French Ministry of Foreign Affairs and the Moroccan Ministry of Higher Education and Scientific Research.

\section{References}

[1] H. T. Nembach, J. M. Shaw, C. T. Boone, and T. J. Silva, "Mode- and size-dependent Landau-Lifshitz damping in magnetic nanostructures: evidence for nonlocal damping," Physical Review Letters, vol. 110, no. 11, Article ID 117201, 2013.

[2] Y. Tserkovnyak, E. M. Hankiewicz, and G. Vignale, "Transverse spin diffusion in ferromagnets," Physical Review B: Condensed Matter and Materials Physics, vol. 79, no. 9, Article ID 094415, 2009.

[3] H. Ammari, L. Halpern, and K. Hamdache, "Asymptotic behaviour of thin ferromagnetic films," Asymptotic Analysis, vol. 24, no. 3-4, pp. 277-294, 2000.
[4] K. Hamdache and M. Tilioua, "On the zero thickness limit of thin ferromagnetic films with surface anisotropy energy," Mathematical Models \& Methods in Applied Sciences, vol. 11, no. 8, pp. 1469-1490, 2001.

[5] M. Kružík and A. Prohl, "Recent developments in the modeling, analysis, and numerics of ferromagnetism," The SIAM Review, vol. 48, no. 3, pp. 439-483, 2006.

[6] A. Visintin, "On the Landau-Lifshitz equation for ferromagnetism," Japan Journal of Applied Mathematics, vol. 2, no. 1, pp. 69-84, 1985.

[7] F. Alouges and A. Soyeur, "On global weak solutions for Landau-Lifshitz equations: existence and nonuniqueness," Nonlinear Analysis. Theory, Methods \& Applications, vol. 18, no. 11, pp. 1071-1084, 1992.

[8] P. Podio-Guidugli and V. Valente, "Existence of global-intime weak solutions to a modified Gilbert equation," Nonlinear Analysis: Theory, Methods \& Applications, vol. 47, no. 1, pp. 147158, 2001.

[9] T. Roubiček, G. Tomassetti, and C. Zanini, "The Gilbert equation with dry-friction-type damping," Journal of Mathematical Analysis and Applications, vol. 355, no. 2, pp. 453-468, 2009.

[10] G. Carbou, M. A. Efendiev, and P. Fabrie, "Relaxed model for the hysteresis in micromagnetism," Proceedings of the Royal Society of Edinburgh: Section A Mathematics, vol. 139, no. 4, pp. 759-773, 2009.

[11] M. Tilioua, "Current-induced magnetization dynamics. Global existence of weak solutions," Journal of Mathematical Analysis and Applications, vol. 373, no. 2, pp. 635-642, 2011.

[12] M. Hadda and M. Tilioua, "On magnetization dynamics with inertial effects," Journal of Engineering Mathematics, vol. 88, pp. 197-206, 2014.

[13] G. Carbou and P. Fabrie, "Time average in micromagnetism," Journal of Differential Equations, vol. 147, no. 2, pp. 383-409, 1998.

[14] M. Langlais and D. Phillips, "Stabilization of solutions of nonlinear and degenerate evolution equations," Nonlinear Analysis. Theory, Methods \& Applications, vol. 9, no. 4, pp. 321-333, 1985.

[15] L. C. Evans, "Partial regularity for stationary harmonic maps into spheres," Archive for Rational Mechanics and Analysis, vol. 116, no. 2, pp. 101-113, 1991.

[16] F. Lin and C. Wang, The Analysis of Harmonic Maps and Their Heat Flows, World Scientific Publishing, Hackensack, NJ, USA, 2008.

[17] B. Guo and S. Ding, Landau-Lifshitz Equations, vol. 1 of Frontiers of Research with the Chinese Academy of Sciences, World Scientific, Hackensack, NJ, USA, 2008.

[18] P.-L. Sulem, C. Sulem, and C. Bardos, "On the continuous limit for a system of classical spins," Communications in Mathematical Physics, vol. 107, no. 3, pp. 431-454, 1986.

[19] F. Alouges and P. Jaisson, "Convergence of a finite element discretization for the Landau-LIFshitz equations in micromagnetism," Mathematical Models \& Methods in Applied Sciences, vol. 16, no. 2, pp. 299-316, 2006.

[20] S. Bartels and A. Prohl, "Convergence of an implicit finite element method for the Landau-LIFshitz-Gilbert equation," SIAM Journal on Numerical Analysis, vol. 44, no. 4, pp. 14051419, 2006.

[21] S. Bartels, J. Ko, and A. Prohl, "Numerical analysis of an explicit approximation scheme for the Landau-LIFshitz-Gilbert equation," Mathematics of Computation, vol. 77, no. 262, pp. 773-788, 2008. 


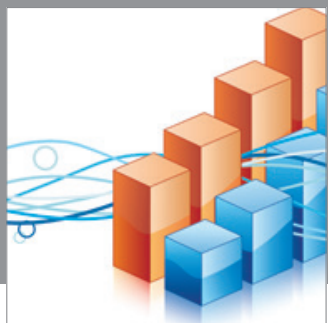

Advances in

Operations Research

mansans

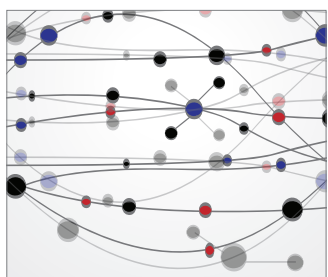

The Scientific World Journal
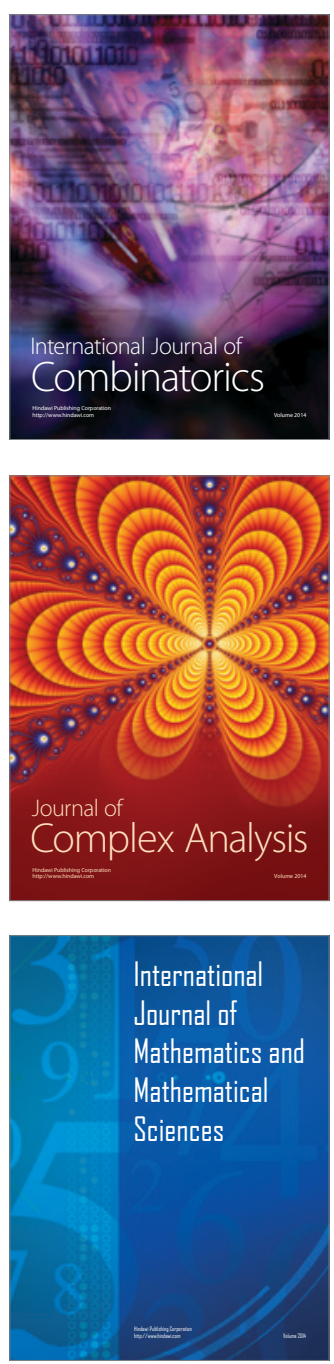
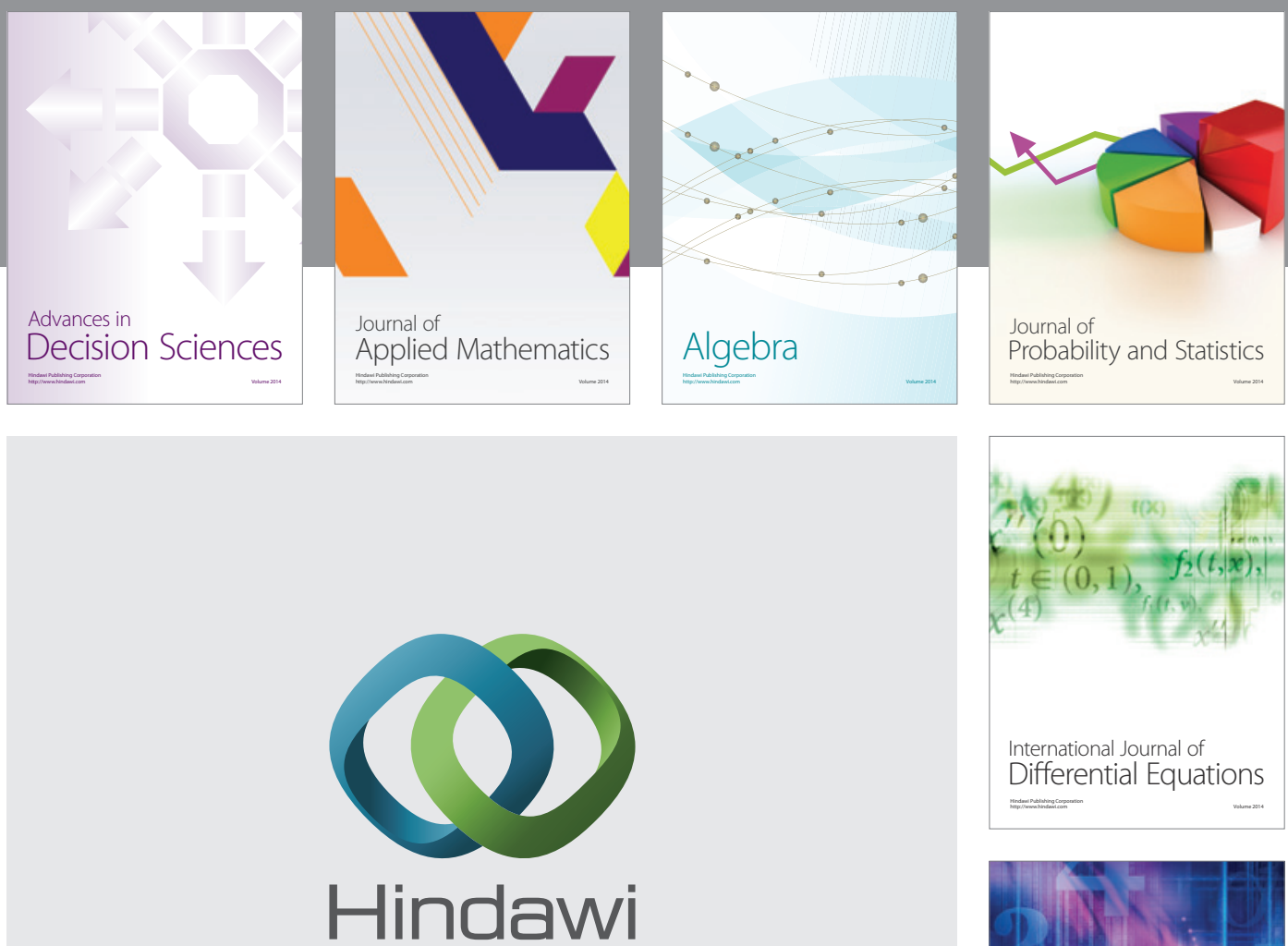

Submit your manuscripts at http://www.hindawi.com
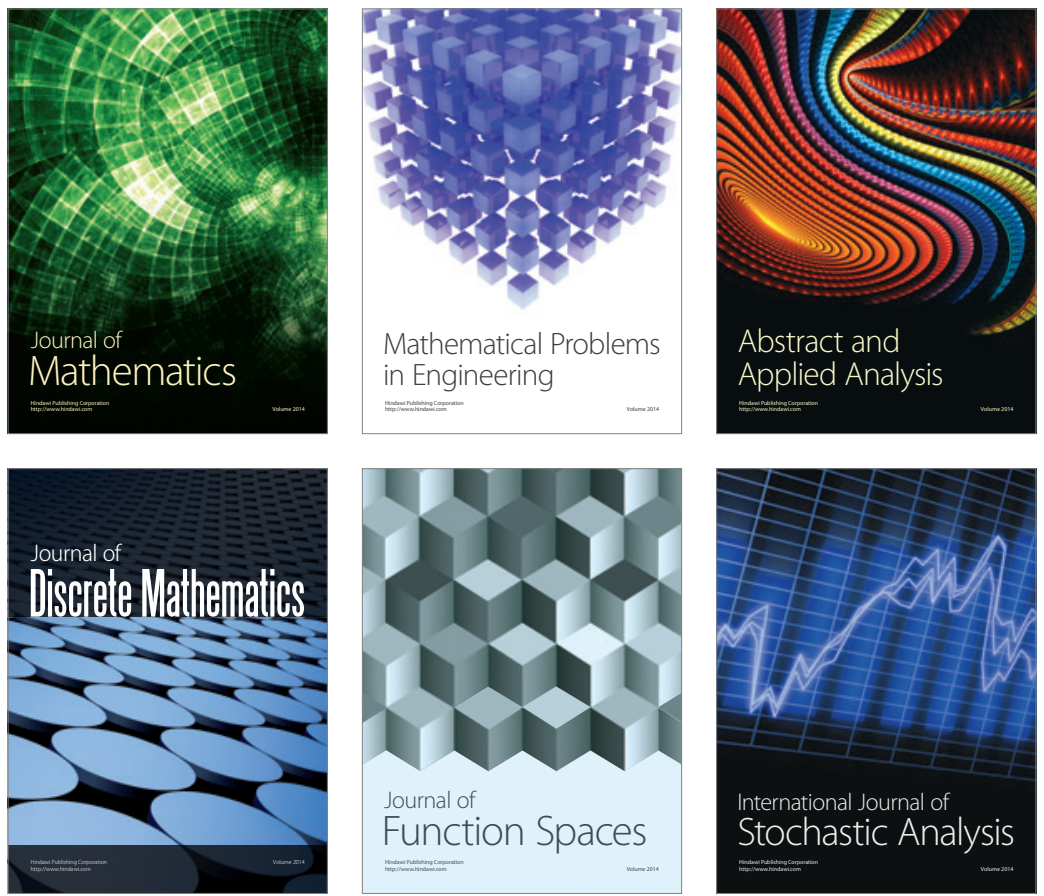

Journal of

Function Spaces

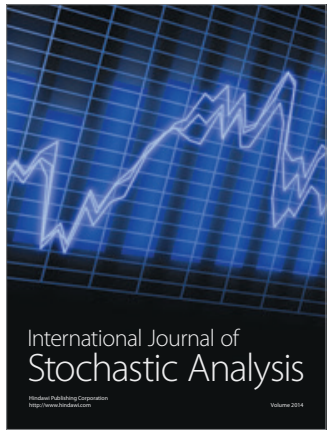

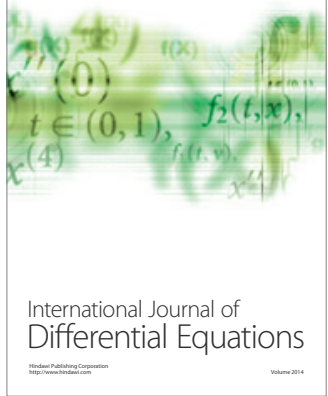
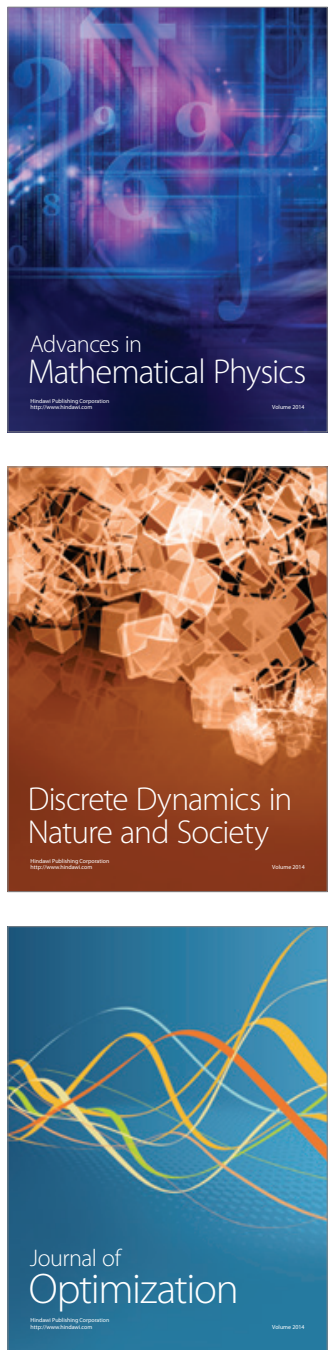